\title{
Two-region model for positive and negative plasma sheaths and its application to Hall thruster metallic anodes
}

\author{
E. Ahedo a) and D. Escobar \\ Escuela Técnica Superior de Ingenieros Aeronáuticos, Universidad Politécnica de Madrid, \\ Plaza Cardenal Cisneros, 28040 Madrid, Spain
}

(Received 19 June 2007; accepted 2 January 2008; published online 24 March 2008)

\begin{abstract}
An asymptotic presheath/sheath model for positive and negative sheaths in front of a conducting electrode, with a continuous parametric transition at the no-sheath case, is presented. Key aspects of the model are as follows: full hydrodynamics of both species in the presheath; a kinetic formulation with a truncated distribution function for the repelled species within the sheath; and the fulfillment of the marginal Bohm condition at the sheath edge, in order to match the two formulations of the repelled species. The sheath regime depends on the ratios of particle fluxes and sound speeds between the two species. The presheath model includes the effect of a magnetic field parallel to the wall on electrons. An asymptotic, parametric study of the anode presheath is carried out in terms of the local ion-to-electron flux ratio and Hall parameter. The drift-diffusive model of magnetized electrons fails in a parametric region that includes parts of the negative sheath regime. In the case of the Hall parameter vanishing near the electrode and a weakly collisional plasma, a quasisonic, quasineutral plateau forms next to the sheath edge. () 2008 American Institute of Physics.
\end{abstract}

[DOI: $10.1063 / 1.2888523$ ]

\section{INTRODUCTION}

\section{A. Hall thruster near-anode region}

There exists a limited understanding of the plasma behavior in the near-anode region of a Hall thruster discharge and its influence on the thruster operation. Experiments and models have centered the research on the ionization and acceleration regions, which seem more relevant for the plasma and thruster responses and are more accessible to direct plasma measurements. Nonetheless, the plasma behavior in the near-anode region is important for the anode heating and the stability of the whole discharge. Zhurin et al., ${ }^{1}$ in a review of the large Russian experience on Hall thrusters, assert that, under normal operation, electron thermal transport is more than sufficient to sustain the discharge, and thus a negative (i.e., electron-repelling) sheath needs to be formed. When the thermal flux is insufficient to conduct the discharge current, a positive (i.e., electron-attracting) sheath develops; this situation arises for low propellant flows and results in the discharge easily becoming extinguished. ${ }^{1}$

Negative sheaths are ion-attracting and result in a noticeable ion backcurrent in the rear part of the chamber. Ion backcurrents (extending up to a $60 \%$ of the channel length) were reported experimentally by Bishaev and $\mathrm{Kim}^{2}$ and Kim. ${ }^{3}$ Direct measurements of the near-anode region have been made recently by Dorf et al. ${ }^{4,5}$ In Ref. 4, positive and negative anode falls are found, depending on (i) the metallic anode being clean or coated by a dielectric film, and (ii) the values of the control parameters (discharge voltage $V_{d}$, mass flow, etc.). For clean anodes, potential falls were negative preferentially and tended to decrease when $V_{d}$ was

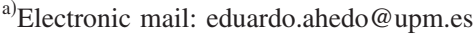

decreased. In Ref. 5, the near-anode region for clean anodes and three different magnetic configurations are investigated. Since the magnetic lines intersect obliquely the anode, the anode potential fall is not uniform. A positive sheath was measured in part of the anode only for a particular magnetic configuration (which presented an intermediate zero magnetic-field point). Since this saddle point was near the exit of the chamber, a direct relation with the behavior of the anode fall is unclear.

The fluid and hybrid(particle-in-cell/fluid) models of Fife, ${ }^{6}$ Ahedo, Martínez-Sánchez, and co-workers, ${ }^{7-9}$ and Barral et al. ${ }^{10}$ assume the existence of a negative sheath and recover the ion backcurrent region of Bishaev-Kim experiments. In agreement with the observations of Ref. 4, these models show that, when $V_{d}$ decreases, the negative anode fall decreases and eventually vanishes. However, Ahedo et al. ${ }^{11}$ pointed out that, for small or zero negative falls, electron inertial effects become relevant in the near-anode region, thus invalidating locally the electron diffusive model (and the resulting Ohm's law) commonly used.

Dorf et al. ${ }^{12,13}$ discussed solutions of the plasma discharge with no sheath at the anode. In Ref. 12, a diffusive (i.e., low axial drift) electron model is used and two regimes for the anode sheath are proposed: a negative fall regime (corresponding to the classical large sheath solution) and a no sheath regime (with a classical thermal flux of electrons to the anode). In Ref. 13, the particular case of a vanishing magnetic field near the anode region is treated in a nonconventional way: instead of using the sheath/presheath asymptotic procedure, the collisionless sheath formalism is extended far beyond the non-neutral plasma region, in order to recover large electron axial drifts.

Ahedo and Rus ${ }^{14}$ analyzed a discharge model with 
partial electron inertia effects (included first by Barral et $a l^{10}{ }^{10}$ ) and the two-regime model of Dorf et al. ${ }^{12}$ for the anode sheath. They demonstrated that, for small negative falls, electron inertia effects bound the azimuthal energy of electrons to values of the order of their internal energy (thus bounding the energy deposition at the anode too). However, the Ahedo-Rus model cannot give solutions with a positive anode sheath. A first reason is that a positive anode sheath regime was not postulated. The second and main reason is that in a positive sheath, the axial electron flux (i.e., perpendicular to the sheath) is expected to be "supersonic" (in the sense given by the appropriate Bohm condition), which means that inertia effects on the electron axial velocity (not included in Ref. 14) cannot be dismissed in the near-anode region.

\section{B. Presheath/sheath models}

This paper presents a two-region (presheath/sheath) asymptotic model of the plasma around a conducting electrode, covering the full parametric range of positive and negative sheath potential falls. Emphasis is put in deriving a formulation valid for small sheaths and obtaining a continuous parametric transition from negative to positive potential falls. Well-known solutions for large sheaths are recovered. A feature of the model (specially suitable for the Hall thruster case) is the inclusion of a magnetic field parallel to the wall, of an intermediate intensity, such that electrons are magnetized but ions remain unmagnetized. Nonetheless, the model applies to an unmagnetized plasma too. The choice of a macroscopic formulation for the presheath is dictated by the aim of analyzing different phenomena (such as magnetic effects, plasma production, collision processes, etc.), which would be hardly tractable with a kinetic formulation.

The case of a large sheath (in front of a dielectric generally) and zero magnetic field (or a magnetic field perpendicular to the wall) corresponds basically to the TonksLangmuir (TL) problem, which is the paradigm of plasma/ wall interaction problems. ${ }^{15}$ The suitability of a macroscopic formulation of the TL presheath, even in the collisionless limit, has been discussed repeatedly; ${ }^{16-18}$ in particular, the comparison of the solution of the TL kinetic model with four fluid models found disagreements in particle and heat fluxes within the range of $10 \% .{ }^{17}$ Presheath solutions for large sheaths and wall-parallel magnetic fields have been formulated macroscopically by Behnel ${ }^{19}$ and Ahedo ${ }^{20}$ and kinetically by Schmitz et al. ${ }^{21}$ Most models for large sheaths consider a Maxwell-Boltzmann equilibrium law for the repelled species, which comes from either a macroscopic force equilibrium or the assumption of a Maxwellian distribution function.

Small sheaths around a dielectric wall in an unmagnetized source plasma, in front of a dielectric wall, were found by Emmert et al. ${ }^{22}$ and Schwager and Birdsall ${ }^{23}$ for (unlikely) large ion-to-electron temperature ratios. Schwager and Birdsall pointed out that a truncated distribution function (e.g., a cutoff Maxwellian distribution) must be used for the repelled species in order to obtain a physically correct solution for small sheaths. Also, they tested their kinetic model with particle-in-cell simulations, finding a good agreement between them. Moderately small negative sheaths are common in dielectric walls with high secondary-electron emission; ${ }^{24,25}$ in this case, the use of a fully Maxwellian distribution instead of a cutoff Maxwellian one leads to errors in heat deposition at the wall of about $20 \%$, for sheath potentials still of the order of the plasma temperature. ${ }^{26}$

A central aspect of the model presented here is the dual treatment of the repelled species, which is modeled macroscopically in the presheath and with a truncated distribution function in the sheath. Notice first that the validity of this procedure is closely related to the agreement between macroscopic and kinetic formulations of the presheath (in the TL problem, for instance). Second, the matching conditions at the presheath/sheath edge become crucial in order to avoid discontinuities in main plasma magnitudes. The fulfillment of the marginal Bohm criterion at the two sides of the sheath edge provides the key for a satisfactory, continuous solution. This matching condition was already used in a presheath/ sheath model for high secondary-electron emission walls where the primary-plus-secondary electron population had also a dual treatment in the two regions. ${ }^{27}$

The layout of the rest of the paper is the following. Section II presents the presheath model and derives an appropriate Bohm condition for any sheath potential. Section III analyzes the sheath model and the matching conditions at the sheath edge. Section IV discusses presheath solutions, mainly in relation with Hall thruster plasmas. Apart from obtaining numerical solutions, attention is given to asymptotic solutions and parametric characterization of the different sheath and presheath regimes. Conclusions are presented in Sec. V. A short Appendix summarizes the derivation of the Bohm condition for a multicomponent plasma from the sheath equations.

\section{PRESHEATH EQUATIONS AND BOHM CONDITION}

Figure 1(a) sketches the two-region, one-dimensional (1D) model around a conducting electrode (hereafter, called anode). The anode A is a perfect collector of ions and electrons and produces negligible secondary particle emission. The two-region formalism, based on the asymptotic limit $\lambda_{D} \ll \ell_{p s}$, with $\lambda_{D}$ the Debye length and $\ell_{p s}$ the smallest spatial scale of the quasineutral plasma, is invoked. Sheath and presheath solutions are obtained independently in their own scales and then matched asymptotically at the sheath edge (called point B). The sheath appears as a surface discontinuity in the presheath model.

The following macroscopic model is considered for the presheath:

$$
\begin{aligned}
& n_{e}=n_{i} \equiv n, \\
& n_{\alpha} u_{z \alpha} \equiv g_{z \alpha}=\text { const, } \quad(\alpha=i, e), \\
& \left(m_{i} u_{z i}^{2}-T_{i}\right) \frac{1}{u_{z i}} \frac{d u_{z i}}{d z}=-e \frac{d \phi}{d z},
\end{aligned}
$$



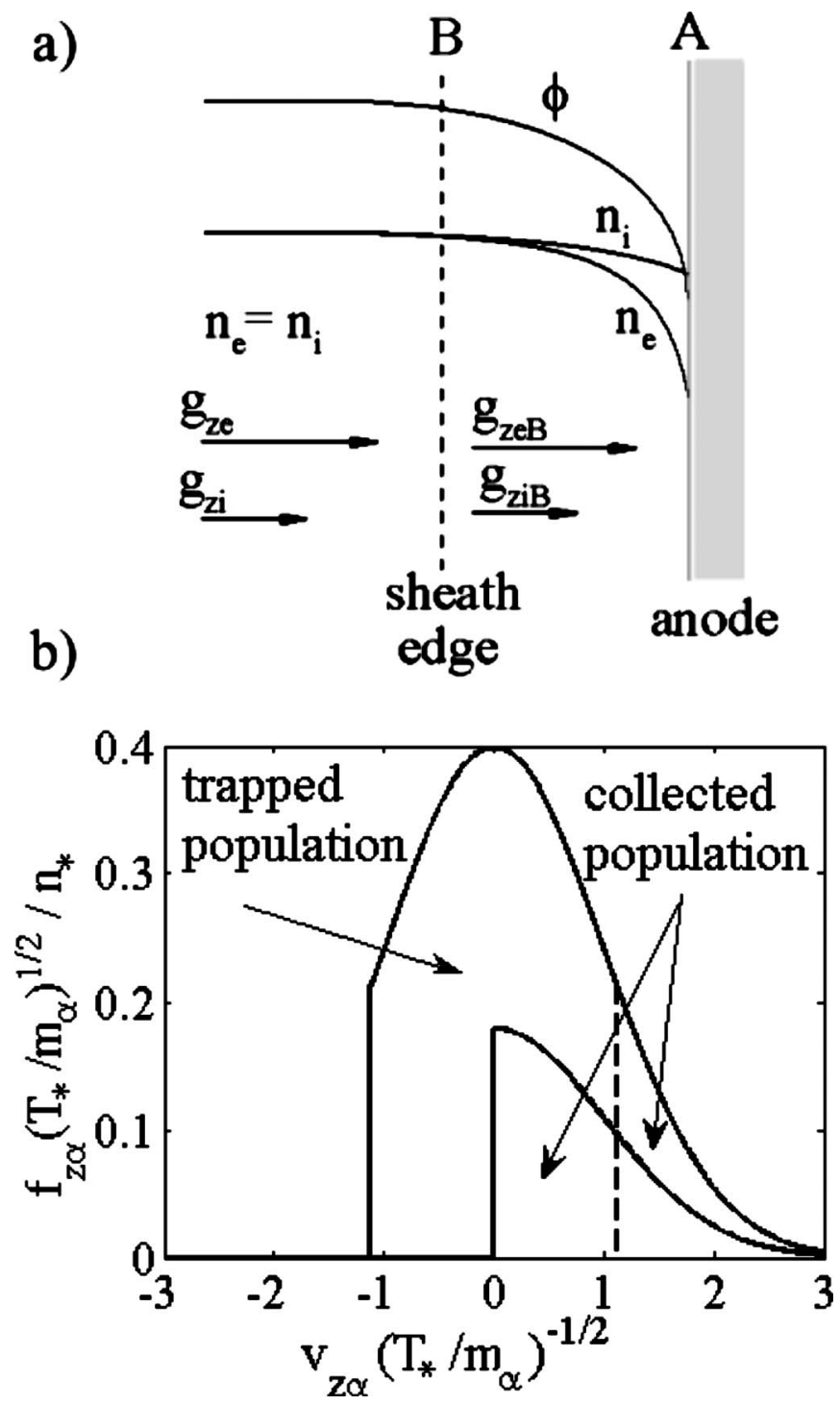

FIG. 1. (a) Sketch of the presheath/sheath model for a negative sheath and a conducing electrode. (b) The truncated Maxwellian distribution function for the repelled species at the sheath edge $\mathrm{B}\left(f_{z \alpha}=0\right.$ for $\left.v_{z \alpha}<-\sqrt{2 e \phi_{A B} / m_{e}}\right)$ and anode $\mathrm{A}\left(f_{z \alpha}=0\right.$ for $\left.v_{z \alpha}<0\right)$, with $\phi_{A B}$ the sheath potential fall.

$$
\begin{aligned}
& \left(m_{e} u_{z e}^{2}-T_{e}\right) \frac{1}{u_{z e}} \frac{d u_{z e}}{d z}=e \frac{d \phi}{d z}+m_{e}\left(\omega_{e} u_{\theta e}-\nu_{e} u_{z e}\right), \\
& u_{z e} \frac{d u_{\theta e}}{d z}=-\omega_{e} u_{z e}-\nu_{e} u_{\theta e},
\end{aligned}
$$

where $n_{\alpha}$ are the species densities, $u_{z i}$ is the ion axial velocity, $u_{z e}$ and $u_{\theta e}$ are the electron (axial and azimuthal) velocities, $\phi$ is the electric potential, $\nu_{e}$ is the momentum-transfer collision frequency, and $\omega_{e}=e B / m_{e}(>0)$ is the electron gyrofrequency of a (nonuniform) magnetic field parallel to the anode. The ion Larmor radius is assumed much larger than the extension of the region under consideration. The introduction of the magnetic field makes the model suitable for the study of the Hall thruster near-anode region. The unmagnetized case $B=0$ is considered too.
For the sake of focusing on the analysis on the main phenomena, density gradients have been assumed larger than temperature gradients, so these last ones can be omitted from Eqs. (3) and (4). Then, equations for the plasma temperatures and heat fluxes, which would complete the model, are not needed here. Alternatively, an adiabatic treatment of any of the two species (say $\alpha$ with $\bar{\gamma}_{\alpha}$ the specific-heat ratio) would result only in substituting $T_{\alpha}$ by $\bar{\gamma}_{\alpha} T_{\alpha}$ in the equations. Also for the sake of centering the discussion, Eq. (2) assumes that the net production of plasma is negligible in the region under study, postponing the discussion of plasma production effects to the end of this section. The ion and electron fluxes into the presheath region, $g_{z \alpha}$, are inputs of this model. In a Hall thruster, the fluxes near the anode are determined by the plasma behavior far from the anode presheath, in the ionization and acceleration regions; for normal operation, one has $g_{z i A} / g_{z e A}<10 \%$ at the anode.

From Eq. (2) and quasineutrality, the two axial velocities are related by

$$
\frac{u_{z i}}{u_{z e}}=\frac{g_{z i B}}{g_{z e B}},
$$

where subscript B refers to values at the sheath edge. From Eqs. (3), (4), and (6), one has

$$
\left[m_{e} u_{z e}^{2}+m_{i} u_{z i}^{2}-\left(T_{e}+T_{i}\right)\right] \frac{1}{u_{z e}} \frac{d u_{z e}}{d z}=m_{e}\left(\omega_{e} u_{\theta e}-\nu_{e} u_{z e}\right)
$$

where, if convenient, $d \ln u_{z e} / d z$ can be substituted by $d \ln u_{z i} / d z$. This equation, which does not contain the ambipolar electric field, shows that the quasineutral plasma, when viewed as a single fluid, is driven by the magnetic and resistive forces on electrons (plasma production and temperature gradients would introduce additional driving forces in this equation). Equations (5)-(7) determine the three velocity components $u_{z i}, u_{z e}$, and $u_{\theta e}$ (for known temperature fields). Since $u_{z e}$ cannot become zero, the only singularity of this set of equations corresponds to

$$
m_{e} u_{z e}^{2}+m_{i} u_{z i}^{2}=T_{e}+T_{i}
$$

This is just a standard sonic condition in the one-fluid formulation of the plasma; $\left(T_{e}+T_{i}\right) n$ and $\left(m_{e} u_{z e}^{2}+m_{i} u_{z i}^{2}\right) n$ are the plasma pressure and axial momentum flux, respectively. If the momentum of one of the plasma species is negligible, Eq. (8) recovers the simple sonic condition for the accelerated species in the two-fluid formulation.

Making the ansatz $\operatorname{sgn} u_{\theta e}=-\operatorname{sgn}\left(\omega_{e} u_{z e}\right)$, the right-hand side of Eq. (7), i.e., the net force driving the plasma, cannot be zero. Hence, the sonic condition (9) leads to a singular point, which can be located only at the boundary of the quasineutral domain, that is, at the sheath edge,

$$
m_{e} u_{z e B}^{2}+m_{i} u_{z i B}^{2}=T_{e B}+T_{i B}
$$

Equation (9) is equivalent to 


$$
\frac{n_{e B}}{T_{e B}-m_{e} u_{z e B}^{2}}+\frac{n_{i B}}{T_{i B}-m_{i} u_{z i B}^{2}}=0,
$$

which is just a particular case of the marginal Bohm condition (A3) for a multicomponent plasma: here both ions and electrons are treated hydrodynamically. Using Eqs. (6) and (9), the "Bohm velocities" of ions and electrons at B satisfy

$$
u_{z i B}^{2}(\gamma)=\frac{T_{B}}{m_{i}} \frac{\gamma^{2}}{1+\gamma^{2}}, \quad u_{z e B}^{2}(\gamma)=\frac{T_{B}}{m_{e}} \frac{1}{1+\gamma^{2}},
$$

where

$$
\gamma=\frac{g_{z i B}}{g_{z e B}} \sqrt{\frac{m_{i}}{m_{e}}}
$$

is the relevant i-e flux ratio parameter of the model, and $T_{B}=T_{e B}+T_{i B}$ is the total plasma temperature.

Observe that, were $d \phi / d z$ an external electric field, Eqs. (3) and (4) would yield that the ion and electron fluxes could present singular points at $u_{z i}=\sqrt{T_{i} / m_{i}}$ and $u_{z e}=\sqrt{T_{e} / m_{e}}$, respectively. However, since the electric field is self-adjusted by the quasineutral plasma, it acts as an additional pressure force that makes the flow regular there. Mathematically, the right-hand sides of Eqs. (3) and (4) are zero; in other words, those singularities are removable. ${ }^{28,29}$ In particular, Eq. (3) states that the electric field is zero at $u_{z i}=\sqrt{T_{i} / m_{i}}$.

The derivatives of plasma magnitudes around point $\mathrm{B}$ satisfy

$$
-\left.\frac{d n_{e}}{d z}\right|_{B},\left.\quad \frac{d u_{z e}}{d z}\right|_{B},\left.\quad \frac{d u_{z i}}{d z}\right|_{B} \rightarrow+\infty,
$$

and

$$
\left.\frac{d \phi}{d z}\right|_{B} \times \operatorname{sgn}\left(m_{i} u_{z i B}^{2}-T_{i B}\right) \rightarrow-\infty .
$$

They are infinite only in the quasineutral scale of the presheath, i.e., asymptotically. In fact, these conditions are indicating the transition to the much smaller spatial scale of the Debye sheath, where most gradients are of the order of $\lambda_{D}^{-1}$. Only the derivative of $u_{\theta e}(z)$ is regular at point $\mathrm{B}$, and $u_{\theta e}$ is going to be constant inside the Debye sheath.

The sign of $d \phi /\left.d z\right|_{B}$ determines whether the sheath is going to be positive or negative. Equation (14) establishes that the transition between the negative- and positive-sheath regimes consists of a single no-sheath case, which is characterized by

$$
\gamma=\sqrt{T_{i B} / T_{e B}}, \quad \text { i.e., } \quad u_{z i B}=c_{i B}, \quad u_{z e B}=c_{e B},
$$

with

$$
c_{\alpha B}=\sqrt{T_{\alpha B} / m_{\alpha}}, \quad(\alpha=i, e)
$$

the species sound speed at the sheath edge. For the no-sheath case, the electric field at the anode is

$$
\left.\frac{e}{m_{e}} \frac{d \phi}{d z}\right|_{B}=-\frac{T_{i B}}{T_{B}}\left(\omega_{e B} u_{\theta e B}-\nu_{e B} u_{z e B}\right),
$$

i.e., the anode is electron-attracting.

The negative sheath regime (NR) is expected for

$$
\gamma>\sqrt{T_{i B} / T_{e B}}, \quad u_{z i B}>c_{i B}, \quad u_{z e B}<c_{e B},
$$

and the positive sheath regime (PR) corresponds to the opposite inequality signs. Even if $T_{i B} \ll T_{e B}$, the ion temperature must be retained in order to reproduce the positive regime. (Hence, the boundary condition $u_{z i B}=0$, used in some Hall thruster models, never yields a correct closure with the anode.)

Bohm velocities in Eq. (11) recover classical expressions in the asymptotic regimes. In the large-negative sheath regime (LNR),

$$
\gamma \gg 1, \quad u_{z i B} \simeq \sqrt{\frac{T_{B}}{m_{i}}}, \quad u_{z e B} \ll \sqrt{\frac{T_{B}}{m_{e}}},
$$

the classical Bohm velocity for ions is found, whereas electrons perform a low-drift, diffusive motion. In the large positive sheath regime (LPR),

$$
\gamma \ll 1, \quad u_{z i B} \ll \sqrt{\frac{T_{B}}{m_{i}}}, \quad u_{z e B} \simeq \sqrt{\frac{T_{B}}{m_{e}}},
$$

the opposite situation arises: the ion velocity is negligible (near the anode), plasma dynamics are dominated by the electron fluid, and the classical Bohm expression for $u_{z e B}$ applies.

Figures 2(a)-2(c) plot the continuous and gentle evolution of $u_{z i B}$ and $u_{z e B}$ from the LNR to the LPR. Figure 2(a) plots the universal function of Eq. (9), and Figs. 2(b) and 2(c) illustrate the dependence on the i-e flux ratio, Eq. (11). These curves are universal except for the location of the single no-sheath case, which depends on $T_{i B} / T_{e B}$, Eq. (15). Dashed lines in Fig. 2(a) depict the Bohm velocities for the asymptotic large-sheath regimes.

\section{A. Plasma production effects}

Let $\nu_{\text {prod }}$ be the plasma net production frequency, resulting from plasma ionization and recombination. Plasma production leads to the inclusion of extra source terms (proportional to $\nu_{\text {prod }}$ ) on the right-hand side of Eqs. (2)-(5). The principal term appears in Eq. (2), which becomes

$$
\frac{d}{d z} n_{\alpha} u_{z \alpha}=n_{e} \nu_{\text {prod }}, \quad \alpha=i, e,
$$

and leads to spatial variations on the axial fluxes of ions and electrons, $g_{z \alpha}$. It is straightforward to check that the resulting set of equations continues to satisfy the same Bohm conditions (9) and (11).

Keeping $\nu_{\text {prod }}$ and dropping the magnetic field, the present macroscopic model of a flowing plasma (with or without plasma production) can be compared to the Schwager-Birdsall version of the Tonks-Langmuir kinetic model. ${ }^{23}$ Indeed, the no-sheath condition (15) can be expressed as

$$
\frac{g_{z i B}}{g_{z e B}}=\frac{c_{i B}}{c_{e B}} \equiv \sqrt{\frac{m_{e} T_{i B}}{m_{i} T_{e B}}},
$$

which generalizes to flowing plasmas and conducting walls, 

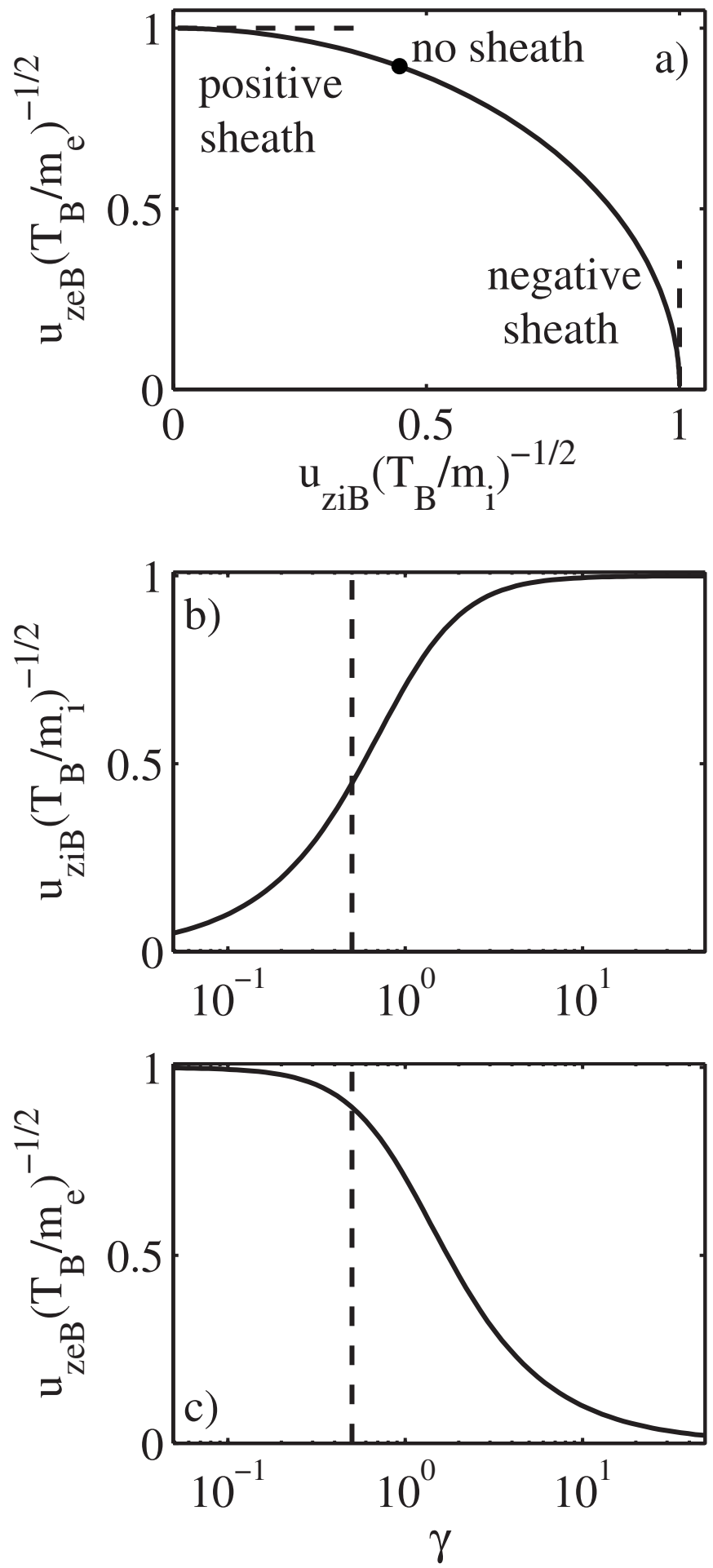

FIG. 2. (a) Relative evolution of ion and electron (axial) velocities at the sheath edge. The asterisk represents the no-sheath transition for $T_{i B} / T_{e B}$ $=0.25$. Dashed lines represent the asymptotic behavior for the large negative and positive regimes. The no-sheath model used in Refs. 12 and 14 corresponds to the horizontal segment $u_{z e} \approx 0.4 \sqrt{T_{B} / m_{e}}$ and $u_{z i}<\sqrt{T_{B} / m_{i}}$. (b) and (c) Evolution of the axial velocities at $\mathrm{B}$ with the i-e flux ratio parameter; dashed lines mark the no-sheath case.

the Schwager-Birdsall no-sheath condition, $T_{i B} / T_{e B}=m_{i} / m_{e}$, for a source plasma and a dielectric wall $\left(g_{z i B}=g_{z e B}\right)$. In their case, an unlikely large ion-to-electron temperature ratio is required for the sheath vanishing. For a conducting electrode and $T_{i B} \leq T_{e B}$, sheath vanishing requires only that the ion-toelectron flow ratio is small (of the order $\sqrt{m_{e} / m_{i}}$ ).

Hence, for a small Debye length, two-species plasma, placed in front of a conducting wall, $g_{z i B} / g_{z e B}$ and $c_{i B} / c_{e B}$ are the only parameters determining whether a negative or positive sheath forms, independently of plasma production, collisional, and magnetic effects in the presheath.

\section{SHEATH SOLUTION}

The conditions at the sheath edge for the NR and PR, Eq. (18), state that the species attracted by the anode potential enters "supersonically" into the sheath, whereas the repelled species presents a "subsonic" axial drift. Hereafter we discuss the solution for a negative sheath. Because of the symmetry between the two sheath types, the solution for a positive sheath will come out from just exchanging the roles of ions and electrons. Observe that the symmetry is not total because of the electron azimuthal velocity, $u_{\theta e}$, but this is constant within the sheath and does not intervene in the sheath equations.

The sheath potential fall affects mainly the flux of the repelled species and requires to know the velocity distribution function of that species at the wall edge. In a dielectric wall, the potential fall allows the plasma to satisfy the zero current condition. In the present model, where the particle fluxes of ions and electrons are determined from the plasma behavior outside the presheath, the role of the potential is to adjust, for the repelled species, the particle flux collected at the wall to the flux coming into the sheath from the quasineutral presheath. Hence a kinetic formulation is mandatory for the repelled species (hereafter, electrons).

Making the ansatz that the sheath potential profile is monotonic, $\phi$ can be used as the spatial variable within the sheath. Let $v_{z e}$ represent the velocity of individual electrons perpendicular to the anode, and $f_{z e}\left(v_{z e}, \phi\right)$ characterize the 1D electron velocity distribution function (VDF), once integral moments on the parallel velocity components have been carried out. Since the sheath is collisionless and unmagnetized, individual electrons conserve their axial energy $\left(m_{e} v_{z e}^{2}+2 e \phi\right)$ within the sheath and $f_{z e}\left(v_{z e}, \phi\right)$ depends only on the VDF at the sheath edge $\mathrm{B}, f_{z e B}\left(v_{z e}\right)$. If $\phi_{A B}$ is the sheath potential fall and the wall is perfectly absorbing and nonemissive, the distribution $f_{z e B}$ distinguishes among three intervals for the electron perpendicular velocity: $v_{z e}<-v_{s h}$ $=\sqrt{2 e \phi_{A B} / m_{e}}$ for electrons emitted by the wall, with $f_{z e B}=0$; $-v_{s h}<v_{z e}<v_{s h}$ for plasma electrons reflected back within the sheath, with $f_{z e B}\left(v_{z e}\right)=f_{z e B}\left(-v_{z e}\right)$; and $v_{z e}>v_{s h}$ for plasma electrons that are collected by the wall, with $f_{z e B}>0$. Next, assuming that electron thermalization is high enough in the presheath, $f_{z e B}$ is approximated by a cutoff Maxwellian distribution (see Refs. 23 and 25, and previous references cited therein). Then, applying the constants of motion, one ends with 


$$
\begin{aligned}
f_{z e}\left(v_{z e}, \phi\right)= & n_{\star} \sqrt{\frac{m_{e}}{2 \pi T_{\star}}} \exp \left(e \frac{\phi-\phi_{B}}{T_{\star}}\right) \\
& \times \exp \left(-\frac{m_{e} v_{z e}^{2}}{2 T_{\star}}\right) H\left(v_{z e}+\sqrt{\frac{2 e\left(\phi-\phi_{A}\right)}{m_{e}}}\right),
\end{aligned}
$$

shown in Fig. 1(b), with $T_{\star}, n_{\star}$, and $\phi_{A B}$ the three parameters characterizing the VDF and $H\left(v_{z}\right)$ the Heaviside step function. The integration of Eq. (23) over $v_{z e}$ yields the electron density $^{13}$

$$
n_{e}(\phi)=n_{\star} \exp \left(\frac{e \phi-e \phi_{B}}{T_{\star}}\right) \frac{1+\operatorname{erf}\left(\sqrt{e\left[\phi-\phi_{A}\right] / T_{\star}}\right)}{2} .
$$

The three parameters that define $f_{z e}$ must be determined from three matching conditions at the sheath/presheath edge, where the two formulations for the repelled species meet. The matching conditions that preserve the main plasma dynamics are (1) the continuity of the electron density,

$$
\int_{-\infty}^{\infty} d v_{z e} f_{z e B} \equiv n_{\star} \frac{1+\operatorname{erf}\left(\sqrt{e \phi_{A B} / T_{\star}}\right)}{2}=n_{e B} ;
$$

(2) the continuity of the electron flux,

$$
\int_{0}^{\infty} d v_{z e} v_{z e} f_{z e A} \equiv n_{\star} \sqrt{\frac{T_{\star}}{2 \pi m_{e}}} \exp \left(-\frac{e \phi_{A B}}{T_{\star}}\right)=g_{z e B} ;
$$

and (3) the fulfillment of the marginal Bohm condition (A3) at the sheath edge side too. Keeping the macroscopic formulation for ions inside the sheath, but using now the equilibrium law (24) for electrons, the Bohm condition on the sheath side becomes

$$
\frac{n_{e B}}{T_{\star}}+\frac{n_{\star} \exp \left(-e \phi_{A B} / T_{\star}\right)}{2 T_{\star} \sqrt{\pi e \phi_{A B} / T_{\star}}}+\frac{n_{i B}}{T_{i B}-m_{i} u_{z i B}^{2}}=0 .
$$

The equivalence between this condition and Eq. (10) on the presheath side yields the third matching condition.

The matching conditions yield $n_{\star}, \phi_{A B}$, and $T_{*}$ in terms of magnitudes of the presheath side $\left(n_{e B}, g_{z e B}\right.$, and $\left.T_{e B}\right)$, thus determining completely the presheath/sheath matching for the repelled species. It is easy to check that the electric charge $\rho(\phi)$ is always monotonic in this two-species plasma. Then, the Poisson equation and the Bohm condition assure that the electric field and potential profiles are monotonic in the sheath, as assumed above. Notice that the profile of the sheath potential is not needed to solve completely the presheath; only the matching conditions are necessary.

The resulting sheath solution recovers the classical "large sheath" case and, at the same time, provides a gentle parametric transition between positive and negative sheaths. For $e \phi_{A B} / T_{\star} \gg 1$, Eqs. (25) and (27) yield $n_{e B} \simeq n_{\star}$ and

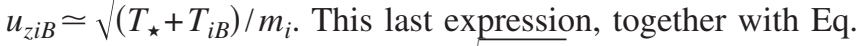
(26) (which assures that $u_{z e B} \ll \sqrt{T_{\star}} / m_{e}$ ) and Eq. (2), yields $T_{e B} \simeq T_{\star}$. Therefore, for $e \phi_{A B} / T_{e B} \gg 1, f_{z e B}\left(v_{z e}\right)$ is a quasifull Maxwellian and

$$
u_{z i B}=\sqrt{\frac{T_{e B}+T_{i B}}{m_{i}}}, \quad u_{z e B}=\sqrt{\frac{T_{e B}}{2 \pi m_{e}}} \exp \left(-\frac{e \phi_{A B}}{T_{e B}}\right) .
$$

These expressions do not apply for moderate and small sheaths. In particular, for $e \phi_{A B} / T_{\star} \ll 1, f_{z e B}$ tends to be a half-Maxwellian, $n_{e B} \simeq n_{\star} / 2, T_{e B} \simeq 2 T_{\star} / \pi$, and $u_{z i B}$ and $u_{z e B}$ approach the no-sheath values of Eq. (15). For positive sheaths, Eqs. (23)-(28) are applicable if subscripts $i$ and $e$ are exchanged, and $\phi_{A B}$ is substituted by $-\phi_{A B}=\left|\phi_{A B}\right|$.

Figure 3(a) shows the variation of the sheath potential drop with the axial drift of the repelled species from the "large sheath" to the "no sheath" cases. It can be observed that the "large sheath" model yields errors larger than $20 \%$ for $\bar{u}_{z \alpha B}=u_{z \alpha B}\left(m_{\alpha} / T_{\alpha B} /\right)^{1 / 2}>0.15$. Figures 3(b) and 3(c) depict the two other constants $\left(n_{*}\right.$ and $\left.T_{*}\right)$ characterizing the cutoff Maxwellian at point $\mathrm{B}$, in terms of $n_{\alpha B}, T_{\alpha B}$, and the drift velocity. Finally, Fig. 3(d) illustrates the gentle evolution of the sheath potential fall with the i-e flux parameter, $\gamma$, from the positive to the negative regime.

The continuity of the Bohm condition across the sheath edge is doubly justified. On the one hand, it is based on the continuity of a dynamic property of the plasma, a sonic plasma flow at the sheath edge (i.e., equal static and dynamic pressures). On the other hand, since $\phi_{A B} \rightarrow 0$ exactly at $\bar{u}_{z \alpha B} \rightarrow 1,(\alpha=i, e)$, it allows a gentle, continuous parametric transition from positive to negative sheaths. This last aspect is not immediate to satisfy. For instance, in Ref. 30, we found that imposing the matching of the electron temperatures instead of the Bohm conditions leads to $\phi_{A B}=0$ for $\bar{u}_{z e B} \approx 0.9$, as shown in Fig. 3(a), and no satisfactory solution can be found for the range $0.9<\bar{u}_{z e B}<1$.

The present model yields a continuous parametric transition at $\phi_{A B}=0$ for the ion and electron fluid velocities. The flux of electron energy at the anode, for the two sheath regimes, is

$$
Q_{z e A}=g_{z e B} \times \begin{cases}2 T_{\star}+\frac{1}{2} m_{e} u_{\theta e B}^{2} & \text { for } \phi_{B}>\phi_{A}, \\ e\left|\phi_{A B}\right|+\frac{5}{2} T_{e B}+\frac{1}{2} m_{e}\left(u_{z e B}^{2}+u_{\theta e B}^{2}\right), & \text { for } \phi_{B}<\phi_{A} .\end{cases}
$$



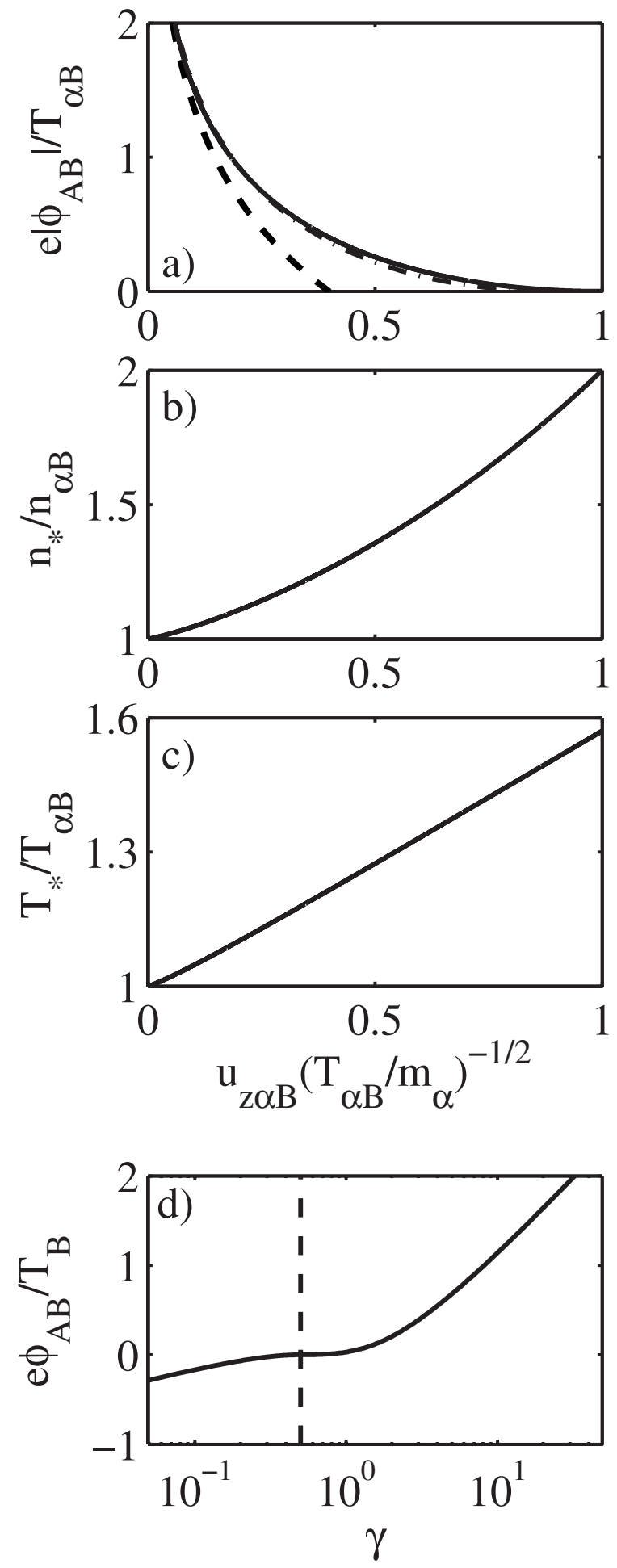

FIG. 3. (a) Sheath potential drop versus the perpendicular macroscopic velocity of the repelled species (subscript $\alpha$ ). The dashed line is the large sheath approximation. The dash-and-dot line corresponds to the matching conditions proposed in Ref. 30. (b) and (c) Constants $n_{\star}$ and $T_{\star}$, defining the cutoff Maxwellian at the sheath edge, $f_{z \alpha B}$, versus the density and temperature at B. (d) Evolution of the sheath potential fall with the i-e flux ratio; the dashed line marks the no-sheath case.

One has that $Q_{z e A} / g_{z e B}-m_{e} u_{\theta e B}^{2} / 2$ is equal to $\pi T_{e B}$ for $\phi_{A B} \rightarrow 0^{+}$and $3 T_{e B}$ for $\phi_{A B} \rightarrow 0^{-}$, thus the difference being a mere $5 \%$ at the positive-to-negative sheath transition, where both expressions meet. Therefore, the matching of the two formulations at the sheath edge is perfect for integral moments of $f_{z e B}$ up to order 1 , and very good for order 2 . Indeed, the agreement is better than the one found between the fluid and kinetic models of the Tonks-Langmuir presheath. ${ }^{17}$

Clearly, a truncated VDF needs to be used for the repelled species in front of a small sheath, and a truncated Maxwellian seems the appropriate choice when particle thermalization is high. For partial thermalization or anisotropic plasmas conditions, the truncated $f_{z e B}$ may differ from Eq. (23), but there is still a wide range of conditions where the procedures and trends seen here will continue to apply.

\section{PRESHEATH SOLUTION}

The presheath equations (1)-(5)_or the more general ones that include plasma production — can be integrated as an initial-value problem, from a "very subsonic" point where $m_{e} u_{z e}^{2}+m_{i} u_{z i}^{2} \ll T_{e}+T_{i}$ to the sheath edge B, where Eq. (9) is satisfied. At the initial point, the azimuthal electron velocity satisfies (under rather general conditions) the diffusive approximation

$$
u_{\theta e}^{d} \simeq-u_{z e} \omega_{e} / \nu_{e} .
$$

The numerical integration is straightforward and some examples are shown below. Therefore, this section is focused on the characterization of the presheath solution in terms of the different parameters and conditions (mainly within the context of Hall thruster plasmas). The preceding sections showed that the (dimensionless) sheath solution depends on the i-e flux parameter $\gamma$ but not on the local Hall parameter at the anode,

$$
\beta=\omega_{e B} / \nu_{e B} .
$$

On the contrary, the presheath equations indicate that its (dimensionless) solution is going to depend on (i) parameters $\gamma$ and $\beta$; (ii) the functional form of $T_{e}(z), T_{i}(z), \omega_{e}(z), \nu_{e}(z)$; and (iii) $\nu_{\text {prod }}$. The characterization of the solution comes mainly from $\gamma$ and $\beta$, so, in order to assess their influence, a "constant-coefficient" model, where $\nu_{\text {prod }}=0$ and the four other functions are constant (near the anode), is considered first.

\section{A. Constant-coefficient model}

Figures 4(a)-4(e) depict profiles of plasma magnitudes in the presheath for several values of $\beta$ and $\gamma$, covering both the negative and positive regimes of the constant model. The electric potential increases upon entering the near-anode presheath, and presents a maximum within the presheath at the location where the ion flux satisfies $u_{z i}=\sqrt{T_{i B} / m_{i}}$, Eq. (3). As $\left|u_{z i B}\right|$ decreases from the value $\sqrt{T_{B} / m_{i}}$ at the large negative regime, the maximum of $\phi(z)$ moves toward the sheath edge $\mathrm{B}$ and disappears eventually in the positive sheath regime. Since the density evolves as $\propto u_{z e}^{-1}$, the density at the sheath edge is very small in the positive regime. Observe that density (i.e., pressure) gradients can be as important as the electric field in order to drive the plasma to sonic conditions at the sheath edge. A relevant feature is that the electron azimuthal velocity becomes larger than the electron thermal velocity already within the negative regime. 

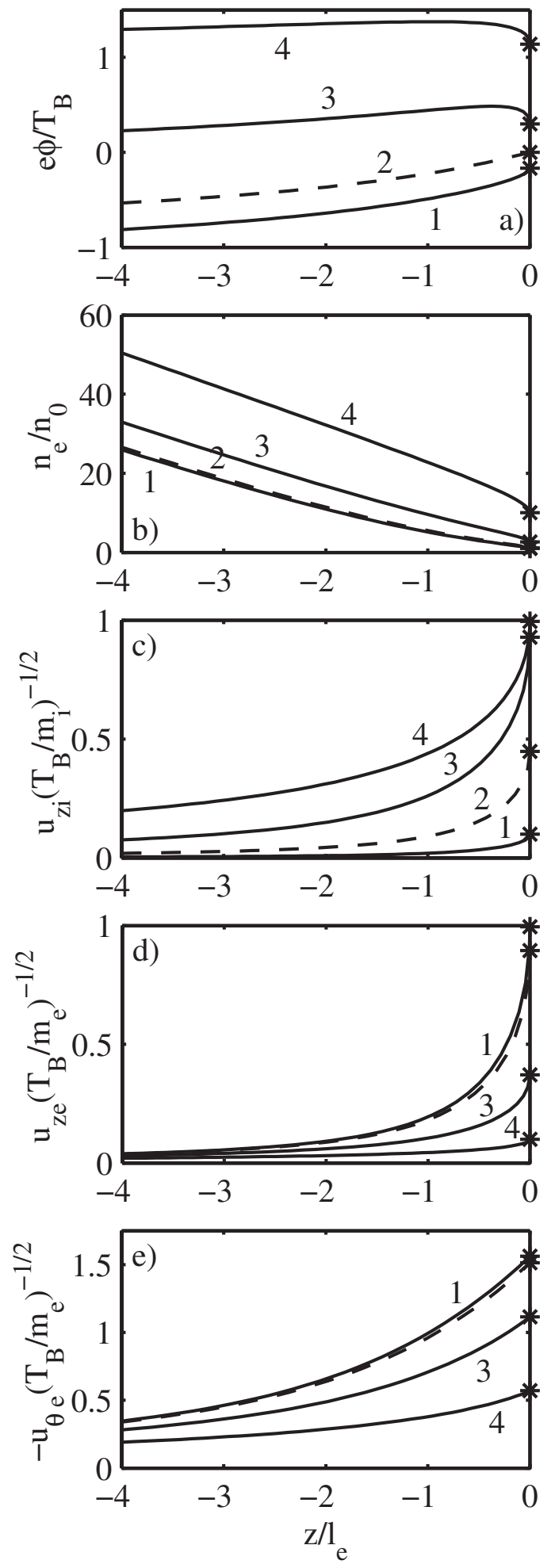

FIG. 4. Plasma profiles in the anode presheath for $T_{i B} / T_{e B}=0.25, \beta=10$, and different values of the i-e flux ratio: $\gamma=0.1(1), 0.5$ (2, no sheath case), 2.5(3), $10(4) ; n_{0}=g_{z e B}\left(m_{e} / T_{B}\right)^{-1 / 2}$. The anode sheath is seen here as a surface discontinuity, asterisks indicating values at the sheath edge B.

Figures 5(a)-5(d) illustrate on the influence of $\gamma$ and $\beta$ on relevant plasma parameters. They complement Figs. 2(b), 2(c), and 3(d). Figure 5(a) plots the azimuthal electron velocity at the sheath edge. It shows that the diffusive approximation (30) holds up to the sheath edge only in the large negative regime. The difference $u_{\theta e}(z)-u_{\theta e}^{d}(z)$ is maximum at
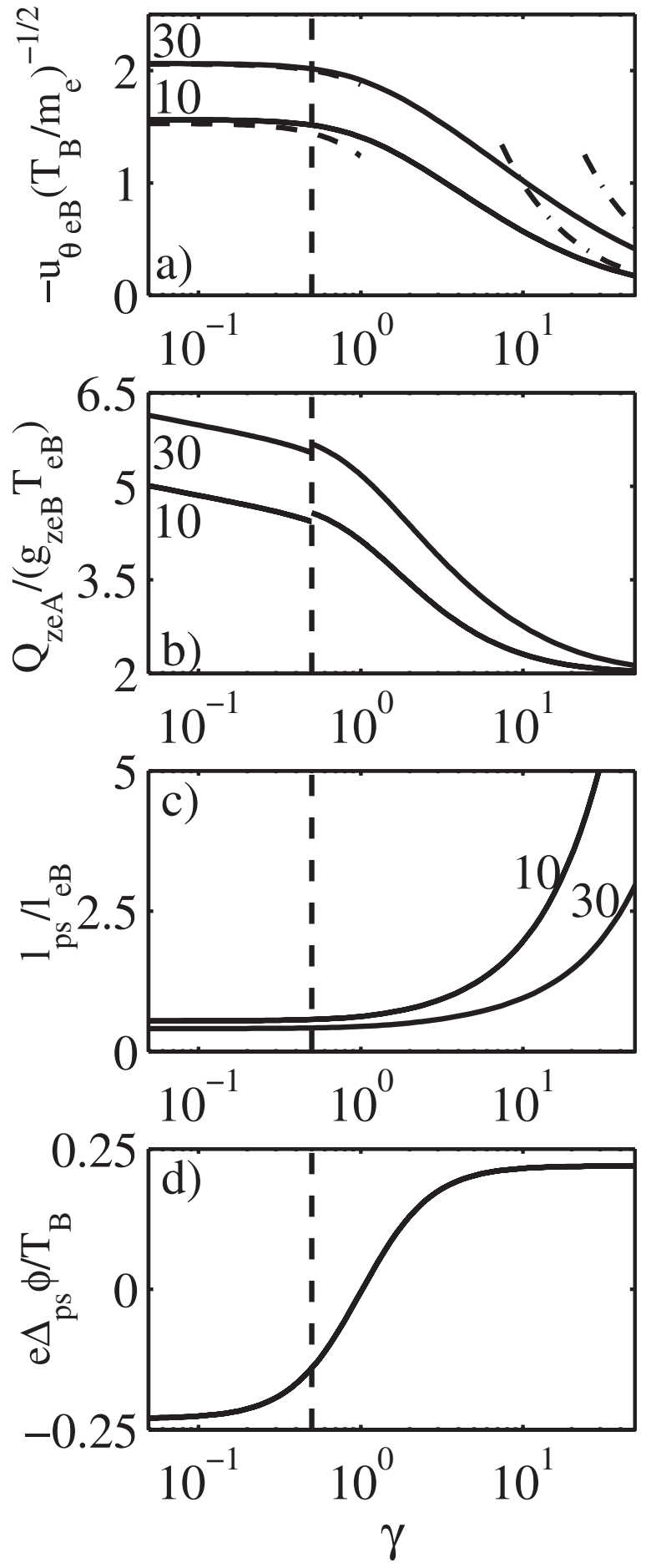

FIG. 5. Evolution of relevant presheath parameters with $\gamma$ for $\beta=10$ and 30 . (a) Electron azimuthal velocity at the sheath edge; dashed-and-dot lines correspond to the two asymptotic regimes. (b) Energy deposited by electrons at the anode, showing a small discontinuity at the positive-to-negative sheath transition, caused by the dual formulation. (c) and (d) Presheath typical thickness and potential fall, measured between the sheath edge $B$ and the point where $\left(m_{e} u_{z e B}^{2}+m_{i} u_{z i B}^{2}\right) / T_{B}=0.1$.

the sheath edge and becomes larger as $\beta$ increases or $\gamma$ decreases. In the positive regime, $u_{\theta e B}$ is bounded by inertia effects to a value of the order of the sound velocity. ${ }^{14}$ This is very positive since it limits effectively the energy deposited by electrons at the anode, Eq. (29) and Fig. 5(b). The relative electron energy flux, $Q_{z e A} / g_{z e B} T_{e B}$, ranges from the classical 
value of 2 for the large negative regime to $\sim 5$ at the regime transition. In the positive regime, $u_{\theta e B}$ is almost constant and the changes of the electron energy flux are due only to $\left|e \phi_{A B}\right|$, which is proportional to $T_{i B}$.

Figures 5(c) and 5(d) show the evolution of the presheath thickness, $\ell_{p s}$, and potential fall, $\Delta_{p s} \phi$, with $\gamma$ and $\beta ; \ell_{e B}=\omega_{e B} \sqrt{T_{B} / m_{e}}$ is the electron gyroradius. The trends of these magnitudes are more important than their values, since the selected "subsonic" bound of the presheath is discretionary. For $T_{i}$ constant, Eq. (3) yields that $e \phi+m_{i} u_{z i}^{2} / 2$ $-T_{i} \ln u_{z i}$ is constant, which explains that $\Delta_{p s} \phi$ depends only on $\gamma$. It is worthwhile to observe that, because of the small changes (in magnitude) of $\Delta \phi_{p s}(\gamma)$, the magnitude $\ell_{p s}^{-1}$ is indeed a measure of the force accelerating the plasma in the presheath. For $\beta$ large, the increase of $\ell_{p s}^{-1}$ as $\gamma$ decreases expresses the fact that a much larger axial electric field is needed to accelerate electrons transversally to the magnetic field in the positive regime than to accelerate the unmagnetized ions in the negative regime.

The exact solutions of Figs. 4 and 5 admit asymptotic solutions in certain parametric regimes. Eliminating the ion velocity $u_{z i}$ in Eq. (7), one has

$$
\left(u_{z e}-\frac{u_{z e B}^{2}}{u_{z e}}\right) \frac{d u_{z e}}{d z}=\frac{\omega_{e} u_{\theta e}-\nu_{e} u_{z e}}{1+\gamma^{2}},
$$

with the electron Bohm velocity $u_{z e B}$, Eq. (11). Equations (31) and (5) for the azimuthal velocity constitute a closed set that admits the first integral

$$
\nu_{e}\left(1+\gamma^{2}\right)\left(u_{z e}+u_{z e B}^{2} / u_{z e}\right)+\omega_{e} u_{\theta e}+\left(\omega_{e}^{2}+\nu_{e}^{2}\right)\left(z-z_{0}\right)=0,
$$

where the constant $z_{0}$ is related to $u_{\theta e B}$ :

$$
\nu_{e}\left(z_{B}-z_{0}\right)\left(1+\beta^{2}\right)=-\beta u_{\theta e B}-2\left(1+\gamma^{2}\right) u_{z e B} .
$$

Substituting $u_{\theta e}$ from Eq. (32) into Eq. (31), a closed equation for $u_{z e}$ is obtained. This can be written as

$$
\left(v-\frac{1}{v}\right) \frac{d v}{d \zeta}=-\sigma\left(\frac{v}{1+\gamma^{2}}+\frac{1}{v}\right)-\zeta
$$

with

$$
\sigma=\sqrt{\frac{1+\gamma^{2}}{1+\beta^{2}}}
$$

$v=u_{z e} / u_{z e B}, \zeta=\left(z-z_{0}\right) \nu_{e} / u_{z e B} \sigma$. The form of the solution of Eq. (34) depends mainly on the new parameter $\sigma$. Figure 6 sketches the distinguished regions and expressions for $\sigma$ in the $(\gamma, \beta)$ parametric plane. If conditions $\gamma \sim 1$ and $\beta \sim 1$ locate the boundaries between the positive and negative regimes, and between magnetized and unmagnetized electron dynamics, respectively, it will be shown below that $\sigma \sim 1$ bounds the cases in which the diffusive approximation (30) holds in (practically) the whole presheath. Observe that $\sigma>1$ covers one part of the negative regime, whereas $\sigma<1$ covers the other part and the whole magnetized, positive regime.

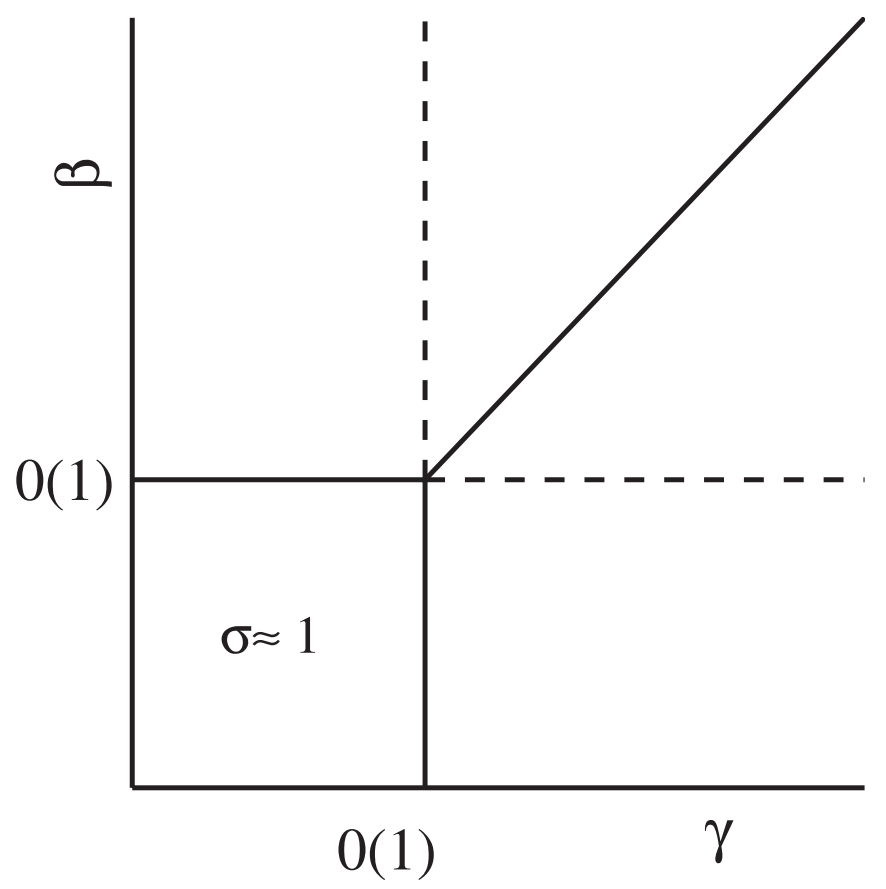

FIG. 6. Sketch of the parametric regions for the different asymptotic regimes of the presheath: $\gamma \gg 1$ for the large negative regime; $\beta \gg 1$ for magnetized electrons; $\sigma \gg 1$ for diffusive electron motion. In the two upper regions, $\sigma \simeq\left(1+\gamma^{2}\right)^{1 / 2} \beta^{-1}$; in the two right-side regions, $\sigma \simeq \gamma\left(1+\beta^{2}\right)^{-1 / 2}$.

An equation very similar to Eq. (34) was analyzed by Ahedo. ${ }^{20}$ Following that analysis, the asymptotic solution of Eq. (34) for $\sigma \ll 1$ is made of a diffusive and a collisionless part:

$$
v(\zeta) \simeq \sigma \exp \left(\zeta^{2} / 2\right) \int_{-\infty}^{\zeta} \exp \left(-u^{2} / 2\right) d u
$$

for $v(\zeta) \ll 1$, and

$$
\zeta=\sqrt{\ln \frac{v^{2}}{2 \pi \sigma^{2}}-v^{2}}
$$

for $v(\zeta) \sim 1$. Particularizing this last expression at the sonic point B and making use of the definition of $\zeta$ and Eq. (33), one has an expression for the azimuthal velocity

$$
\frac{-u_{\theta e B}}{u_{z e B}} \frac{\beta}{\left(1+\beta^{2}\right) \sigma} \simeq \sqrt{\ln \frac{1}{\sigma^{2}}-\ln 2 \pi-1}+2 \sigma,
$$

or, more conveniently,

$$
\frac{-u_{\theta e B}}{\sqrt{T_{B} / m_{e}}} \simeq \sqrt{\ln \frac{\beta^{2}}{1+\gamma^{2}}-\ln 2 \pi-1}+2 \frac{\sqrt{1+\gamma^{2}}}{\beta}>1 .
$$

Since the logarithmic term inside the square root is not very large in most cases, the contribution of the other terms on the right-hand side must be retained.

Equation (34) is not appropriate to solve the case $\sigma \gg 1$. Considering that $\sigma \gg 1$ implies that $\gamma \gg 1$ and making the ansatz that the diffusive approximation (30) applies in the whole presheath, the convenient equation for $\sigma \gg 1$ is 


$$
\left(\frac{T_{B}}{m_{i} u_{z i}^{2}}-1\right) \frac{d u_{z i}}{d z} \simeq \nu_{e B} \sqrt{\frac{m_{e}}{m_{i}}} \frac{\beta^{2}+1}{\gamma},
$$

which is immediate to integrate. The diffusive approximation requires that $\omega_{e}^{-1} d u_{\theta e} d z \ll 1$, which, using Eq. (39), is equivalent to $\sigma \gg 1$. This justifies the ansatz and demonstrates that $\sigma=O(1)$ (in Fig. 6) bounds the asymptotic region where the diffusive limit for electrons applies. For $\sigma \gg 1$, the azimuthal velocity at point $\mathrm{B}$ satisfies

$$
\frac{-u_{\theta e B}}{\sqrt{T_{B} / m_{e}}} \simeq \frac{\beta}{\gamma} \ll 1 .
$$

The two asymptotic expressions, Eqs. (38) and (40), fit very well with the exact solution of Fig. 5(a), and yield $-u_{\theta e B}$ $\sim \sqrt{T_{B} / m_{e}}$ at $\sigma \sim 1$.

Also, from the two asymptotic solutions, the presheath thickness scales as

$$
\frac{\ell_{p s}}{\ell_{e B}} \sim\left(\ln \frac{\beta^{2}}{1+\gamma^{2}}\right)^{-1 / 2}<1
$$

for $\sigma \ll 1$, and

$$
\frac{\ell_{p s}}{\ell_{e B}} \sim \frac{\beta \gamma}{\beta^{2}+1} \gg 1
$$

for $\sigma \gg 1$. The scaling of $\ell_{p s}$ with $\gamma$ and $\beta$ corresponds to the behavior observed in Fig. 5(c).

\section{B. Low magnetic field case}

The above presheath analysis and solutions are valid as long as $\ell_{p s}$ is smaller than the gradient lengths associated to $T_{e}(z), T_{i}(z), \omega_{e}(z)$, and $\nu_{e}(z)$. The presheath thickness is largest for $\sigma \gg 1$, that is, for the (locally) unmagnetized, negative regime, $\beta \ll 1$ and $\gamma \gg 1$. Hence, we focus now on this regime. The study case we choose consists on a Hall parameter decaying within the presheath and becoming negligible at the anode. For the rest of the coefficients, we take $\gamma \gg 1$, and $T_{i}(z), T_{e}(z)$, and $\nu_{e}(z)$ constant.

The relevant equation is Eq. (39) substituting $\beta$ by $\omega_{e}(z) / \nu_{e B}$. First, this equation yields that the generalization of expression (42) for the presheath extension is

$$
\begin{aligned}
\int_{0}^{\ell_{p s}}\left(\frac{\omega_{e}^{2}}{\nu_{e B}^{2}}+1\right) d\left(z_{B}-z\right) & \simeq \gamma \lambda_{e B}\left[\frac{c_{s B}}{u_{z i}}+\frac{u_{z i}}{c_{s B}}\right]_{u_{z i}=c_{i}}^{u_{z i} \ll c_{i}} \\
& \sim \gamma \lambda_{e B},
\end{aligned}
$$

with $c_{s B}=\sqrt{T_{B} / m_{i}}$ the conventional plasma sound speed, and $\lambda_{e B}=\nu_{e B}^{-1} \sqrt{T_{B} / m_{e}}$ the electron collision mean-free path (which in a Hall thruster is often larger than the chamber length). Let $\ell_{1}$ be the extension of the region with $\omega_{e} / \nu_{e B} \ll 1$ and $\ell_{2}$ be the gradient length associated to $\omega_{e}(z)$ in the region with $\omega_{e}(z) / \nu_{e B}>1$, that is, i.e., $\ell_{2} \sim \nu_{e B} d z / d \omega_{e} \ll \lambda_{e B}$. Then, the presheath thickness scales as

$$
\ell_{p s} \sim \ell_{1}+\sqrt[3]{\gamma \lambda_{e B} \ell_{2}^{2}},
$$

and the presheath is constituted by two quasineutral subregions: an unmagnetized plateau of length $\ell_{1}$, where the plasma, driven by the small resistive force, is quasisonic; and

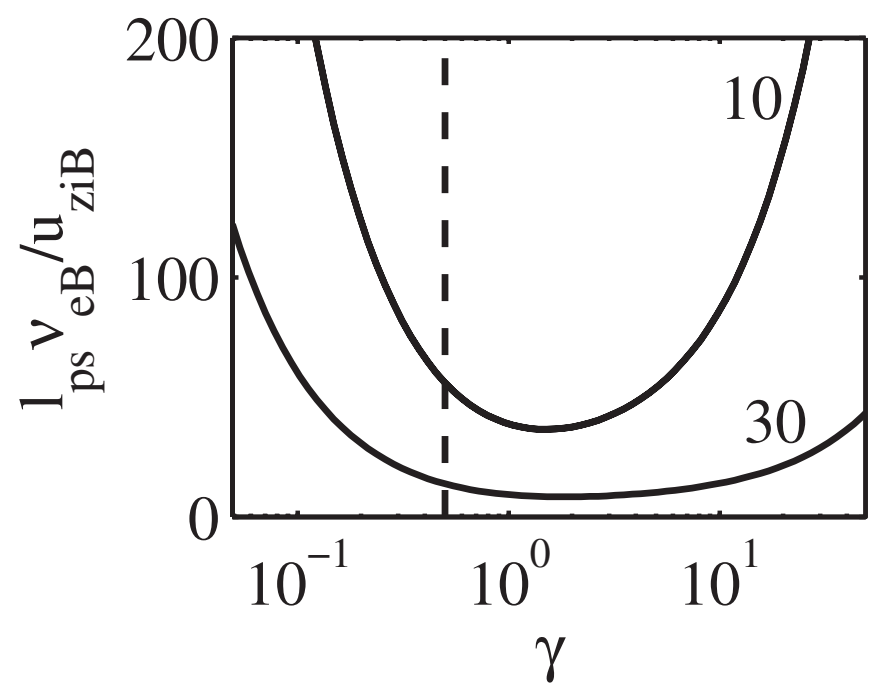

FIG. 7. Dimensionless parameter for the evaluation of plasma production effects in the anode presheath, according to Eq. (45). Same parameters as in Fig. 5.

the magnetized, subsonic subregion with a typical Hall parameter $\omega_{e} / \nu_{e B} \sim\left(\gamma \lambda_{e B} / \ell_{2}\right)^{1 / 3} \gg 1$.

A (locally) unmagnetized near-anode region of a Hall thruster was the subject of the second theoretical paper of Dorf et al. ${ }^{13}$ There, instead of applying the standard sheath/ presheath formalism with its asymptotically exact matching at the sheath edge, they use the sheath (non-neutral) equations for determining both the sheath and the quasineutral plateau. These were then patched with the magnetized subregion.

\section{Plasma production effects}

Since $g_{i z B} \ll g_{e z B}$, plasma production affects mainly the ion flux. According to Eq. (21), plasma production is negligible in the anode presheath as long as

$$
\frac{\ell_{p s} \nu_{e B}}{u_{z i B}} \ll \frac{\nu_{e B}}{\nu_{p r o d}} .
$$

The collision ratio on the right-hand side depends strongly on the electron temperature; it is $\sim 80$ for xenon, $T_{e B}=5 \mathrm{eV}$, and negligible plasma recombination. The left-hand side of Eq. (45) is plotted in Fig. 7 for the same cases as Fig. 5. It shows that plasma production in the presheath can be relevant for small and large values of the i-e flux ratio. In the first case, this is because $g_{i z B}$ is small and thus very sensitive to changes; in the second one, it is because of the large presheath extension. As a general rule, ionization tends to reduce the effective value of $\gamma$ and therefore the presheath extension.

\section{CONCLUSION}

The model presented here determines the presheath/ sheath structure for positive and negative sheaths in front of a metallic electrode and a flowing plasma. A continuous parametric transition at the no-sheath point is achieved, except for a $5 \%$ difference in heat fluxes. The key aspects of 
the model are as follows: first, a macroscopic presheath formulation with full dynamics for both species; second, a kinetic formulation with a truncated velocity distribution function for the repelled species within the sheath; and third, the fulfillment of the marginal Bohm condition at both sides of the presheath/sheath edge, in order to match there the macroscopic magnitudes obtained from the two formulations of the repelled species. The dual treatment is validated by the very small difference in heat fluxes, which is indeed smaller than the differences between macroscopic and kinetic formulations of the Tonks-Langmuir presheath.

The location of the no-sheath point (and thus the sheath regime) depends only on the ratios of particle fluxes and sound speeds of ion and electron species, and it is independent of magnetic effects, plasma ionization, or collision processes. This conclusion, supported by a macroscopic formulation, recovers and extends the no-sheath condition of the kinetic model of Schwager-Birdsall for a source plasma in front of a dielectric wall.

The presheath model was formulated specifically for (simplified) plasma conditions near a Hall thruster anode. The presheath solution depends basically on two parameters: the i-e flux ratio (which decides between negative and positive sheath regimes) and the local Hall parameter (which determines whether electrons are magnetized or not near the anode). These two parameters define a third one-called $\sigma$, Eq. (35)-which characterizes the presheath regime. The presheath solution has been obtained analytically in two asymptotic ranges. First, a conventional electron diffusive model holds only for a parametric subregion of the magnetized, negative regime (corresponding to $\sigma \gg 1$ ). Second, for magnetized electrons and moderate-to-low i-e flux ratios (corresponding to $\sigma \ll 1$ ), the azimuthal electron velocity is of the order of the electron thermal energy, and spatial gradients are large in order to drive electrons perpendicularly to the magnetic field into sonic conditions at the sheath edge.

As the local Hall parameter decreases and the i-e flux ratio increases, the presheath thickness increases, thus making relevant the spatial variation of certain plasma parameters. A case of interest in certain Hall thrusters is that of the Hall parameter vanishing in the near-anode region. In this case, the anode presheath is constituted by an unmagnetized, quasisonic subregion followed by the magnetized subregion. Finally, the influence of plasma production in the presheath has been assessed: first, production does not affect to the positive/negative regime of the sheath; and second, the parametric range for plasma production to reduce the presheath extension has been identified.

The present model provides insight into the plasma behavior in the near-anode region of a Hall thruster but says nothing about the influence of the parameters governing the Hall thruster discharge (i.e., magnetic field topology, discharge voltage, mass flow rate) on the i-e flux ratio near the anode and, as a consequence, on the regime of the anode sheath. From existing models of the Hall thruster full discharge (strictly valid only for large negative sheaths and electron diffusive motion), the i-e flux ratio is known to decrease when ${ }^{31,32}$ the discharge voltage is decreased, the magnetic field is increased, the magnetic field shape is more flat, or the turbulent diffusion decreases. This last case would agree with the assertion of Ref. 5 that the turbulent diffusion is lower for the magnetic field configuration yielding a positive anode fall. A model of the full Hall thruster discharge, which includes all electron inertia terms (and, therefore, the negative-to-positive transition), faces numerical difficulties that have not been solved yet, even in the one-dimensional formulation. Furthermore, in relation to Hall thrusters, this study provides mainly a better insight into the basic physics of the near-anode plasma. In practice, the near-anode region is highly two-dimensional, with nonuniform fluxes to the different anode locations and, more significantly, magnetic field lines impinging at different angles into the anode and connecting it with the lateral dielectric walls.

\section{ACKNOWLEDGMENTS}

This work was sponsored by the Ministerio de Educación y Ciencia of Spain, under Project ESP2007-62694, and by the Air Force Office of Scientific Research, Air Force Material Command, USAF, under Grant No. FA8655-06-13032.

\section{APPENDIX: THE POISSON EQUATION AND THE BOHM CONDITION}

The electrostatic potential in a thin, collisionless sheath satisfies Poisson's equation

$$
\frac{d^{2} \phi}{d z^{2}}=-\frac{\rho(\phi)}{\varepsilon_{0}}, \quad \rho(\phi)=\sum_{j} q_{j} n_{j}(\phi),
$$

where subindex $j$ stands for different species of a multicomponent plasma, and $q_{j}$ for its electric charge. The Bohm condition at the (asymptotic) sheath edge $B$ is

$$
\left.\frac{d \rho}{d \phi}\right|_{B^{+}} \leq 0
$$

and stems from imposing that the non-neutral solution of Poisson's equation that starts from B corresponds to a locally monotonic potential (i.e., it dismisses oscillatory solutions). ${ }^{33}$ In general, when the plasma in the presheath is stationary and "subsonic" (in an appropriate sense), the marginal form (i.e., equality sign) of the Bohm condition applies.

The expressions for $n_{j}(\phi)$ in $\rho(\phi)$ depend on the formulation used for species $j$. Considering that the species density is derived from either an equilibrium law-generally obtained from its velocity distribution function-or a hydrodynamic formulation (subindexes $k$ and $h$, respectively), the Bohm condition can be expressed as ${ }^{34}$

$$
\left.\sum_{k} q_{k}^{2} \frac{d n_{k}}{d\left(q_{k} \phi\right)}\right|_{B}-\left.\sum_{h} \frac{q_{h}^{2} n_{h}}{\bar{\gamma}_{h} T_{h}-m_{h} u_{h}^{2}}\right|_{B} \leq 0,
$$

where $m_{h}$ is particle mass, $u_{h}$ and $T_{h}$ are fluid velocity and temperature, and $\bar{\gamma}_{h}$ depends on heat transfer conditions. For instance, the equilibrium law for a cutoff Maxwellian distribution is Eq. (24). A very relevant fact is that the MaxwellBoltzmann equilibrium law emanates from both kinetic and hydrodynamic formulations. 
Finally, we remind that the potential profile in the sheath comes out from a standard double integration of Eq. (A1), assuming quasineutrality and a negligible electric field at the sheath edge. First, one has

$$
\left(\frac{d \hat{\phi}}{d \chi}\right)^{2}=2 \int_{\hat{\phi}}^{\hat{\phi}_{B}} \hat{\rho}(\hat{\phi}) d \hat{\phi},
$$

where $\quad \chi=\left(z-z_{A}\right) / \lambda_{D}, \quad \lambda_{D}=\sqrt{\epsilon_{0} T_{e B} / e^{2} n_{e B}}, \quad \hat{\phi}=e \phi / T_{e}, \quad \hat{\rho}$ $=\rho / e n_{e B}$, and $d \hat{\phi} /\left.d \chi\right|_{B} \simeq 0$. Then, the integration of Eq. (A4) yields the sheath potential profile in the form $\chi(\hat{\phi})$.

${ }^{1}$ V. Zhurin, H. Kaufman, and R. Robinson, Plasma Sources Sci. Technol. 8, R1 (1999).

${ }^{2}$ A. Bishaev and V. Kim, Sov. Phys. Tech. Phys. 23, 1055 (1978).

${ }^{3}$ V. Kim, J. Propul. Power 14, 736 (1998).

${ }^{4}$ L. Dorf, Y. Raitses, and N. Fisch, J. Appl. Phys. 97, 103309 (2005).

${ }^{5}$ L. Dorf, Y. Raitses, and N. J. Fisch, Phys. Plasmas 13, 057104 (2006).

${ }^{6}$ J. M. Fife, "Hybrid-PIC modeling and electrostatic probe survey of Hall thrusters," Ph.D. thesis, Massachusetts Institute of Technology (1998).

${ }^{7}$ E. Ahedo and M. Martínez-Sánchez, in Proceedings of the 34th Joint Propulsion Conference, Cleveland, paper AIAA-98-8788 (American Institute of Aeronautics and Astronautics, Washington, DC, 1998).

${ }^{8}$ E. Ahedo, J. Gallardo, and M. Martínez-Sánchez, Phys. Plasmas 10, 3397 (2003).

${ }^{9}$ F. Parra, E. Ahedo, M. Fife, and M. Martínez-Sánchez, J. Appl. Phys. 100, 023304 (2006).

${ }^{10}$ S. Barral, K. Makowski, Z. Peradzynski, N. Gascon, and M. Dudeck, Phys. Plasmas 10, 4137 (2003).
${ }^{11}$ E. Ahedo, J. Gallardo, and M. Martínez-Sánchez, Phys. Plasmas 9, 4061 (2002).

${ }^{12}$ L. Dorf, V. Semenov, Y. Raitses, and N. Fisch, in Proceedings of the 38th Joint Propulsion Conference, Indianapolis, paper AIAA 2002-4246 (American Institute of Aeronautics and Astronautics, Washington, DC, 2002).

${ }^{13}$ L. Dorf, V. Semenov, and Y. Raitses, Appl. Phys. Lett. 83, 2551 (2003).

${ }^{14}$ E. Ahedo and J. Rus, J. Appl. Phys. 98, 043306 (2005).

${ }^{15}$ L. Tonks and I. Langmuir, Phys. Rev. 34, 876 (1929).

${ }^{16}$ G. Kino and E. Shaw, Phys. Fluids 9, 587 (1966).

${ }^{17}$ R. Bissell, P. Johnson, and P. Stangeby, Phys. Fluids B 1, 1133 (1989).

${ }^{18}$ J. Scheuer and G. Emmert, Phys. Fluids B 2, 445 (1990).

${ }^{19}$ K. Riemann, Phys. Plasmas 1, 552 (1994).

${ }^{20}$ E. Ahedo, Phys. Plasmas 4, 4419 (1997).

${ }^{21}$ H. Schmitz, K. Riemann, and T. Daube, Phys. Plasmas 3, 2486 (1996).

${ }^{22}$ G. Emmert, R. Wieland, A. Mense, and J. Davidson, Phys. Fluids 23, 803 (1980).

${ }^{23}$ L. Schwager and C. Birdsall, Phys. Fluids B 2, 1057 (1990).

${ }^{24}$ G. Hobbs and J. Wesson, Plasma Phys. 9, 85 (1967).

${ }^{25}$ L. Schwager, Phys. Fluids B 5, 631 (1993).

${ }^{26}$ E. Ahedo and V. de Pablo, Phys. Plasmas 14, 083501 (2007).

${ }^{27}$ E. Ahedo, Phys. Plasmas 9, 4340 (2002).

${ }^{28}$ R. Franklin, Plasma Phenomena in Gas Discharges (Clarendon Press, Oxford, 1976).

${ }^{29}$ H. Valentini, J. Phys. D 21, 311 (1988).

${ }^{30}$ D. Escobar, E. Ahedo, and F. I. Parra, in Proceedings of the 29th International Electric Propulsion Conference, Princeton, NJ, paper IEPC-2005041 (Electric Rocket Propulsion Society, Fairview Park, OH, 2005).

${ }^{31}$ E. Ahedo, P. Martínez-Cerezo, and M. Martínez-Sánchez, in SP-465: 3rd Spacecraft Propulsion Conference, Cannes (France) (European Space Agency, Noordwijk, The Netherlands, 2000), pp. 323-330.

${ }^{32}$ E. Ahedo and D. Escobar, J. Appl. Phys. 96, 983 (2004).

${ }^{33}$ E. Harrison and W. Thompson, Proc. Phys. Soc. London 74, 145 (1959).

${ }^{34}$ K. Riemann, IEEE Trans. Plasma Sci. 23, 709 (1995). 